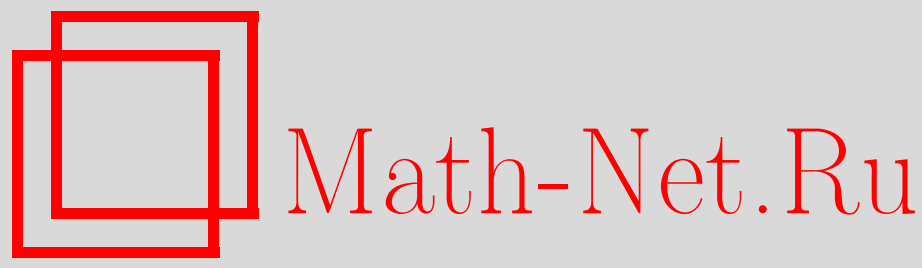

Н. Н. Боголюбов (мл.), А. В. Солдатов, Алгебраические аспекты динамики квантовых многоуровневых систем в методе проекционного оператора, ТМФ, 2018, том 194, номер 2, 259-276

DOI: https://doi.org/10.4213/tmf9395

Использование Общероссийского математического портала Math-Net.Ru подразумевает, что вы прочитали и согласны с пользовательским соглашением http://www . mathnet.ru/rus/agreement

Параметры загрузки:

IP : 54.84 .234 .179

26 апреля 2023 г., 02:58:23

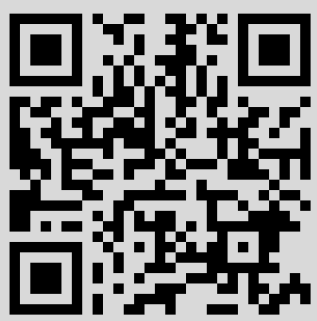




\title{
ФИЗИКА
}

Том 194, № 2

февраль, 2018

(C) 2018 г. Н. Н. Боголюбов (мл.) ${ }^{*}$ А. В. Солдатов*

\section{АЛГЕБРАИЧЕСКИЕ АСПЕКТЫ ДИНАМИКИ КВАНТОВЫХ МНОГОУРОВНЕВЫХ СИСТЕМ В МЕТОДЕ ПРОЕКЦИОННОГО ОПЕРАТОРА}

\begin{abstract}
Методом проекционного оператора получены приближенные локальные и нелокальные по времени управляющие уравнения для редуцированного статистического оператора многоуровневой квантовой системы с конечным числом $N$ собственных квантовых состояний, взаимодействующей одновременно с произвольными внешними классическими полями и диссипативным окружением. Показано, что структура полученных уравнений существенно упрощается, если свободная гамильтонова динамика многоуровневой системы под воздействием внешних полей, а также ее марковская и немарковская эволюции, обусловленные взаимодействием с окружением, описываются посредством представления многоуровневой системы в терминах алгебры $S U(N)$, что позволяет реализовать эффективные численные алгоритмы решения полученных уравнений при исследовании реальных проблем в разнообразных областях теоретической и прикладной физики.
\end{abstract}

Ключевые слова: многоуровневая квантовая система, сокращенное описание, проекционный оператор, открытая система, генератор унитарной группы, операторная алгебра, управляющее уравнение, разложение по системе ортогональных многочленов.

DOI: https://doi.org/10.4213/tmf9395

\section{1. ВВЕДЕНИЕ}

Хорошо известно, что подавляющее большинство практически полезных физических систем являются системами с очень большим и, как правило, даже бесконечным числом степеней свободы. Как следствие, исследование свойств и поведения таких систем составляет предмет неравновесной статистической механики. По этой причине неравновесная статистическая механика превратилась в один из наиболее активно развивающихся разделов теоретической и математической физики, причем полученные в этой области общие теоретические результаты оказывают быстрое и непосредственное влияние не только на многочисленные экспериментальные

* Математический институт им. В.А. Стеклова Российской академии наук, Москва, Россия. E-mail: nikolai_bogolubov@hotmail.com, soldatov@mi.ras.ru 
и теоретические исследования в области прикладной физики, но и на междисциплинарные исследования во многих областях естественных наук. К настоящему времени разработано большое число подходов к исследованию неравновесных классических и квантовых физических систем, из которых можно выделить совокупность методов, чья сущность состоит в том, что в них изначально не ставится задача получения исчерпывающей информации о неравновесном поведении системы путем решения полученных из основополагающих принципов соответствующих классических или квантовых уравнений, описывающих ее динамику, т. е. от полноты описания неравновесной физической системы в терминах полного статистического оператора или функции распределения вероятности намеренно отказываются в пользу исследования поведения только тех степеней свободы или величин, которые характеризуют основные наиболее важные аспекты эволюции системы. Таким образом, следование этой концепции в любом из ее вариантов по определению приводит к так называемому сокращенному описанию физической системы.

Важную, основополагающую роль в развитии этого подхода сыграли ранние работы Боголюбова [1]-[3]. Значительный вклад в развитие важного варианта сокращенного описания, основанного на обобщении фундаментальной идеи статистических ансамблей Гиббса применительно к неравновесным системам, внесли также работы школы Зубарева [4], в которых был сформулирован общий метод неравновесного статистического оператора, нашедший впоследствии приложения в исследовании неравновесных процессов в разнообразных физических системах. Со временем идея сокращенного описания претерпела существенное развитие, что, по мере осмысления и сопоставления использованных при решении конкретных задач в различных областях частных методов и полученных результатов, позволило в конечном итоге концептуально объединить многочисленные разрозненные способы сокращенного описания качественно различных физических систем в рамках единого математического формализма, известного под названием метода проектирования в теории неравновесных процессов, или метода проекционного оператора. История развития как концепции сокращенного описания в целом, так и метода проекционного оператора в частности подробно изложена, например, в монографиях [4]-[6].

На сегодняшний день метод проекционного оператора является одним из основных инструментов исследования неравновесных физических систем при решении важных с прикладной точки зрения задач, что обуславливает актуальность разработки математических методов, которые позволили бы получать в рамках этого общего подхода практически полезные аналитические и численные результаты для конкретных реалистичных и достаточно сложных моделей физических систем. $\mathrm{K}$ таким системам относится, в частности, очень широкий класс квантовых систем с конечномерным гильбертовым пространством состояний. В различных областях физики модели таких систем обычно формулируются в терминах квантовой системы с конечным числом собственных энергетических состояний, взаимодействующей как с внешними полями, так и с диссипативным окружением, стохастическим или обладающим бесконечным числом степеней свободы. В настоящей работе предлагается подход, основанный на общих алгебраических динамических свойствах произвольной квантовой системы со сколь угодно большим, но конечным числом собственных энергетических состояний, позволяющий получать для таких систем разнообразные локальные и нелокальные по времени управляющие уравнения методом проекционного оператора в удобной для последующего исследования их решений методами численного анализа форме. 


\section{2. ДИНАМИКА МНОГОУРОВНЕВОЙ КВАНТОВОЙ СИСТЕМЫ ВО ВНЕШНИХ КЛАССИЧЕСКИХ ПОЛЯХ}

2.1. Динамика $N$-уровневой квантовой системы и алгебра $S U(N)$. Рассмотрим многоуровневую квантовую систему $(S)$, взаимодействующую с внешними полями, которая описывается в общем случае гамильтонианом вида

$$
\widehat{H}_{S}(t)=\sum_{m}^{N} E_{m} \hat{\sigma}_{m m}+\sum_{m, n}^{N} V_{m n}(t) \hat{\sigma}_{m n},
$$

где $N$ - число энергетических уровней системы, а операторы $\hat{\sigma}_{m n}=|m\rangle\langle n|$ представлены в терминах бра- и кет-векторов состояний системы. Эти операторы удовлетворяют коммутационным соотношениям

$$
\left[\hat{\sigma}_{i j}, \hat{\sigma}_{k l}\right]=\hat{\sigma}_{i l} \delta_{j k}-\hat{\sigma}_{k j} \delta_{i l}
$$

Воздействие внешних полей учитывается посредством множителей $V_{m n}(t)$. Динамика системы определяется уравнением Лиувилля-фон Неймана для статистического оператора

$$
\frac{\partial}{\partial t} \hat{\rho}(t)=-\frac{i}{\hbar}\left[\widehat{H}_{S}(t), \hat{\rho}(t)\right]
$$

Ввиду соотношения

$$
\left\langle\hat{\sigma}_{m n}(t)\right\rangle=\operatorname{Sp}\left\{\hat{\sigma}_{m n} \hat{\rho}(t)\right\}=\rho_{m n}(t)
$$

уравнение (3) эквивалентно системе уравнений Гейзенберга для операторов $\hat{\sigma}_{m n}$. Динамическая квантовая система (1) может обладать разнообразными интегралами движения. В частности, в работе [7] было показано, что суммы произведений $q$ матричных элементов статистического оператора вида

$$
C(N, q)=\rho_{j k}(t) \rho_{k l}(t) \ldots \rho_{n j}(t)
$$

где $q=1,2, \ldots, N$ и подразумевается суммирование по всем повторяющимся индексам, не зависят от времени. Первая из этих сумм $C(N, 1)$ представляет собой известный закон сохранения вероятности, т. е. $\operatorname{Sp}\{\hat{\rho}(t)\}=1$. Остальные суммы $C(N, q)$ соответствуют нетривиальным сохраняющимся величинам. Важно отметить, что эти величины сохраняются для произвольных внешних воздействий $V_{m n}(t)$. В принципе, можно рассматривать сами матричные элементы $\rho_{m n}(t)$ как динамические переменные, полностью описывающие эволюцию системы. Тогда величины $C(N, q)$ можно рассматривать как интегралы движения. Существование таких интегралов имеет большое практическое значение, поскольку, как показано в статье [7], они накладывают существенное ограничение на возможность изменения квантового состояния (как чистого, так и смешанного) многоуровневой системы посредством воздействия на нее внешними полями.

В работе [7] были также введены три группы новых эрмитовых операторных переменных $u, v$ и $w$ :

$$
\begin{gathered}
\hat{u}_{j k}=\hat{\sigma}_{j k}+\hat{\sigma}_{k j}, \quad \hat{v}_{j k}=-i\left(\hat{\sigma}_{j k}-\hat{\sigma}_{k j}\right), \quad 1 \leqslant j<k \leqslant N, \\
\hat{w}_{l}=-\left(\frac{2}{l(l+1)}\right)^{1 / 2}\left(\hat{\sigma}_{11}+\cdots+\hat{\sigma}_{l l}-l \hat{\sigma}_{l+1, l+1}\right), \quad 1 \leqslant l \leqslant N-1,
\end{gathered}
$$


так что всего имеется $N^{2}-1$ переменных. Эти операторы являются генераторами алгебры $S U(N)$, т. е. можно показать, что если определить вектор $\hat{\mathbf{s}}$ как упорядоченную последовательность введенных операторных переменных

$$
\hat{\mathbf{s}}=\left(\hat{u}_{12}, \ldots, \hat{u}_{N-1, N}, \hat{v}_{12}, \ldots, \hat{v}_{N-1, N}, \hat{w}_{1}, \ldots, \hat{w}_{N-1}\right),
$$

то его компоненты $\hat{s}_{i}$ удовлетворяют коммутационным соотношениям

$$
\left[\hat{s}_{j}, \hat{s}_{k}\right]=2 i \sum_{l=1}^{N^{2}-1} f_{j k l} \hat{s}_{l}
$$

где по повторяющемуся индексу проводится суммирование от 1 до $N^{2}-1$, a $f_{j k l}-$ полностью антисимметричная структурная постоянная группы $S U(N)$.

В терминах компонент вектора $\hat{\mathbf{s}}$ статистический оператор и гамильтониан системы (1) имеют вид

$$
\begin{gathered}
\hat{\rho}(t)=N^{-1} \hat{I}+\frac{1}{2} \sum_{j=1}^{N^{2}-1} S_{j}(t) \hat{s}_{j} \\
\widehat{H}_{S}(t)=\frac{\hbar}{2} \sum_{j=1}^{N^{2}-1} \Gamma_{j}(t) \hat{s}_{j},
\end{gathered}
$$

где коэффициенты $S_{j}(t)$ и $\Gamma_{j}(t)$ определены как

$$
\begin{aligned}
S_{j}(t) & =\operatorname{Sp}\left\{\hat{\rho}(t) \hat{s}_{j}\right\}, \\
\Gamma_{j}(t) & =\hbar^{-1} \operatorname{Sp}\left\{\widehat{H}_{S}(t) \hat{s}_{j}\right\} .
\end{aligned}
$$

Выражения (10)-(13) получены на основе тождества

$$
\operatorname{Sp}\left\{\hat{s}_{j} \hat{s}_{k}\right\}=2 \delta_{j k}
$$

которое выполняется для любого числа уровней системы $N$. Это же тождество можно использовать для вычисления структурных постоянных $f_{j k l}$. Домножая соотношение (9) на произвольную компоненту $\hat{s}_{p}$ и вычисляя след от правых и левых частей, получаем, что

$$
\operatorname{Sp}\left\{\hat{s}_{j} \hat{s}_{k} \hat{s}_{p}\right\}-\operatorname{Sp}\left\{\hat{s}_{k} \hat{s}_{j} \hat{s}_{p}\right\}=2 i \sum_{l=1}^{N^{2}-1} f_{j k l} \operatorname{Sp}\left\{\hat{s}_{l} \hat{s}_{p}\right\}=4 i f_{j k p}
$$

откуда

$$
f_{j k p}=\frac{i}{4}\left(\operatorname{Sp}\left\{\hat{s}_{k} \hat{s}_{j} \hat{s}_{p}\right\}-\operatorname{Sp}\left\{\hat{s}_{j} \hat{s}_{k} \hat{s}_{p}\right\}\right)
$$

Строго говоря, в разложении для гамильтониана (11) должно присутствовать еще и слагаемое вида $C(t) \hat{I}$, где

$$
C(t)=N^{-1} \operatorname{Sp}\left\{\widehat{H}_{S}(t)\right\}
$$

но это слагаемое не влияет на эволюцию квантовой системы по причине пропорциональности единичному оператору $\hat{I}$ и им можно пренебречь. Тогда из уравнения (3) 
и тождества (14) можно получить систему обыкновенных дифференциальных уравнений непосредственно для компонент вектора коэффициентов $\mathbf{S}$ в виде

$$
\dot{S}_{i}=\sum_{j, k=1}^{N^{2}-1} f_{i j k} \Gamma_{j}(t) S_{k}
$$

Все компоненты вектора $\mathbf{S}$ вещественны, таким образом, уравнения (18) описывают вращение некоторого вектора в пространстве $N^{2}-1$ измерений. Благодаря антисимметричности $f_{i j k}$ длина вектора $\mathbf{S}$ сохраняется и выражается через постоянную движения $C(N, 2)$. В частном случае двухуровневой системы уравнения (18) сводятся к системе уравнений Блоха, описывающих вращение вектора псевдоспина в трехмерном пространстве. Отметим, что все операторы $\hat{s}_{j}$ бесследовые, т. е. $\operatorname{Sp}\left\{\hat{s}_{j}\right\}=0$, поэтому условие сохранения вероятности для статистического оператора $\hat{\rho}(t)$ в представлении (10) выполняется автоматически при любых значениях коэффициентов $S_{j}(t)$, что может оказаться полезным с точки зрения использования каких-либо приближенных методов вычисления этих коэффициентов при исследовании реальных проблем.

Рассмотрим теперь систему уравнений движения Гейзенберга для операторных компонент $\hat{s}_{l}(t)$

$$
\frac{\partial}{\partial t} \hat{s}_{l}(t)=\frac{i}{\hbar}\left[\widehat{H}_{S}(t), \hat{s}_{l}(t)\right]
$$

решения которой всегда можно представить в виде

$$
\hat{s}_{l}(t)=\sum_{p=1}^{N^{2}-1} C_{l p}(t) \hat{s}_{p}, \quad C_{l p}\left(t_{0}\right)=\delta_{l p} .
$$

Отсюда и из (9) и (11) следует система уравнений для коэффициентов $C_{l p}(t)$,

$$
\dot{C}_{l p}(t)=-\sum_{j, k=1}^{N^{2}-1} f_{p j k} \Gamma_{j}(t) C_{l k}(t),
$$

аналогичная для фиксированного индекса $l$ системе уравнений для коэффициентов матрицы плотности $S_{i}(t)$ и вместе с этим описывающая вращение вектора с компонентами $C_{l p}(t)$ в пространстве $N^{2}-1$ измерений. Можно также представить коэффициенты $C_{l p}(t)$ в виде

$$
C_{l p}(t)=\frac{1}{2} \operatorname{Sp}\left(\hat{s}_{l}(t) \hat{s}_{p}\right)=\operatorname{Sp}\left(\hat{s}_{l}(t) \hat{\rho}^{(p)}\left(t_{0}\right)\right)=\operatorname{Sp}\left(\hat{s}_{l} \hat{\rho}^{(p)}(t)\right)=S_{l}^{(p)}(t),
$$

где

$$
\hat{\rho}^{(p)}(t)=N^{-1} \hat{I}+\frac{1}{2} \sum_{j=1}^{N^{2}-1} S_{j}^{(p)}(t) \hat{s}_{j}
$$

есть решение уравнения Лиувилля-фон Неймана для статистического оператора с начальным условием

$$
\hat{\rho}^{(p)}\left(t_{0}\right)=N^{-1} \hat{I}+\frac{1}{2} \hat{s}_{p}
$$


что эквивалентно решению системы уравнений

$$
\dot{S}_{i}^{(p)}=\sum_{j, k=1}^{N^{2}-1} f_{i j k} \Gamma_{j} S_{k}^{(p)}
$$

с начальным условием $S_{i}^{(p)}\left(t_{0}\right)=\delta_{i p}$.

2.2. Динамика $N$-уровневой квантовой системы и бозонное представление алгебры $S U(N)$. Некоторым относительным недостатком системы уравнений (18) является то обстоятельство, что число уравнений растет с ростом числа уровней системы как $N^{2}-1$. Однако для конкретной задачи иногда может оказаться полезным и эффективным способ, позволяющий существенно сократить число уравнений. Заметим, что любая алгебра $S U(N)$ есть частный случай более общей алгебры $S U(N, M)$, а именно $S U(N) \equiv S U(N, 0)$. Алгебра $S U(N, 0)$ допускает представление в терминах бозе-операторов $\left\{\hat{a}_{k}, \hat{a}_{k}^{+}, k=1, \ldots, N\right\}$, которые удовлетворяют коммутационным соотношениям

$$
\left[\hat{a}_{k}, \hat{a}_{l}^{+}\right]=\delta_{k l}, \quad\left[\hat{a}_{k}, \hat{a}_{l}\right]=\left[\hat{a}_{k}^{\dagger}, \hat{a}_{l}^{\dagger}\right]=0 .
$$

Множество всех $N^{2}$ билинейных произведений этих операторов вида

$$
\hat{X}_{j k}=\hat{a}_{j}^{+} \hat{a}_{k}
$$

генерирует алгебру $S U(N, 0)$. Эти операторы коммутируют с так называемым эффективным оператором числа частиц

$$
\hat{N}=\sum_{i=1}^{N} \hat{a}_{i}^{+} \hat{a}_{i}
$$

так что число независимых генераторов алгебры $S U(N, 0)$ равно $N^{2}-1$. Тогда, как показано в работах [8], [9], для любой операторнозначной функции $\widehat{F}\left(\left\{\hat{a}_{i}^{\dagger}(t)\right\},\left\{\hat{a}_{j}(t)\right\}\right)$, $i, j=1, \ldots, N$, вычисление унитарного преобразования

$$
\widehat{F}\left(\left\{\hat{a}_{i}^{\dagger}(t)\right\},\left\{\hat{a}_{j}(t)\right\}\right)=\exp \left(\frac{i}{\hbar} \widehat{H}_{(N, 0)} t\right) \widehat{F}\left(\left\{\hat{a}_{i}^{\dagger}\right\},\left\{\hat{a}_{j}\right\}\right) \exp \left(-\frac{i}{\hbar} \widehat{H}_{(N, 0)} t\right)
$$

с генератором вида

$$
\widehat{H}_{(N, 0)}=\sum_{i, j=1}^{N} \alpha_{i j} \hat{a}_{i}^{+} a_{j}
$$

сводится к нахождению решений уравнений

$$
\frac{d}{d t} \hat{a}_{i}(t)=-\frac{i}{\hbar} \sum_{j=1}^{N} \alpha_{i j} \hat{a}_{j}(t)
$$

описывающих эволюцию бозе-операторов; эти уравнения тривиально обобщаются на случай зависящего явно от времени генератора

$$
\widehat{H}_{(N, 0)}(t)=\sum_{i, j=1}^{N} \alpha_{i j}(t) \hat{a}_{i}^{+} a_{j}
$$


и записываются как

$$
\frac{d}{d t} \hat{a}_{i}(t)=-\frac{i}{\hbar} \sum_{j=1}^{N} \alpha_{i j}(t) \hat{a}_{j}(t)
$$

что соответствует унитарному преобразованию

$$
\widehat{F}(t)=\stackrel{\leftarrow}{T} \exp \left(\frac{i}{\hbar} \int_{t_{0}}^{t} d t^{\prime} \widehat{H}_{(N, 0)}\left(t^{\prime}\right)\right) \widehat{F} \vec{T} \exp \left(-\frac{i}{\hbar} \int_{t_{0}}^{t} d t^{\prime} \widehat{H}_{(N, 0)}\left(t^{\prime}\right)\right)
$$

где символы $\stackrel{\leftarrow}{T}$ и $\vec{T}$ показывают направление возрастания временного аргумента при упорядочении операторных сомножителей по времени при разложении экспоненциальных функций в ряд. Каждому оператору $\hat{\sigma}_{i j}$ можно поставить в соответствие оператор $\widehat{X}_{i j}$ так, что

$$
\hat{\sigma}_{i j} \rightarrow \hat{a}_{i}^{+} \hat{a}_{j} .
$$

Как следствие, операторы $\hat{s}_{k}$ представимы в виде билинейных форм бозе-операторов $\hat{a}_{i}^{\dagger}, \hat{a}_{i}$, и для вычисления динамики $N$-уровневой квантовой системы вместо $N^{2}-1$ уравнений (18) достаточно найти решение $N$ линейных уравнений (33) для $N$ бозеоператоров $\hat{a}_{i}$.

\section{3. КОНВОЛЮТИВНЫЕ И НЕКОНВОЛЮТИВНЫЕ УПРАВЛЯЮЩИЕ УРАВНЕНИЯ В МЕТОДЕ ПРОЕКЦИОННОГО ОПЕРАТОРА}

\section{1. Конволютивное управляющее уравнение для релевантной части} статистического оператора. В работе [10] была предложена удобная универсальная формальная схема построения эволюционных уравнений для редуцированного (или релевантного) статистического оператора, который описывает поведение только тех степеней свободы полной квантовой системы, эволюция которых представляет интерес. Предположим, что полная квантовая ситема описывается гамильтонианом

$$
\widehat{H}(t)=\widehat{H}_{0}(t)+\widehat{H}_{1}(t)
$$

так что удобно сразу перейти в представление взаимодействия

$$
\begin{aligned}
\tilde{\hat{\rho}}(t) & =U_{0}^{+}\left(t, t_{0}\right) \hat{\rho}(t) U_{0}\left(t, t_{0}\right), \\
\widetilde{\widehat{H}}_{1}(t) & =U_{0}^{+}\left(t, t_{0}\right) \widehat{H}_{1}(t) U_{0}\left(t, t_{0}\right),
\end{aligned}
$$

где эволюционный оператор имеет вид

$$
U_{0}\left(t, t_{0}\right)=\stackrel{\leftarrow}{\operatorname{Texp}}\left\{-\frac{i}{\hbar} \int_{t_{0}}^{t} d t^{\prime} \widehat{H}_{0}\left(t^{\prime}\right)\right\}, \quad U_{0}^{+}\left(t, t_{0}\right)=\vec{T} \exp \left\{\frac{i}{\hbar} \int_{t_{0}}^{t} d t^{\prime} \widehat{H}_{0}\left(t^{\prime}\right)\right\}
$$

и обладает обычными свойствами

$$
\begin{aligned}
U_{0}\left(t, t_{0}\right) U_{0}^{+}\left(t, t_{0}\right) & =1, & U\left(t_{1}, t_{2}\right) U\left(t_{2}, t_{3}\right) & =U\left(t_{1}, t_{3}\right), \\
U\left(t, t_{0}\right) U\left(t_{0}, t\right) & =1, & U\left(t_{0}, t\right) & =U^{+}\left(t, t_{0}\right) .
\end{aligned}
$$


Тогда статистический оператор полной квантовой системы удовлетворяет уравнению Лиувилля-фон Неймана

$$
\frac{\partial}{\partial t} \tilde{\hat{\rho}}(t)=-\frac{i}{\hbar}\left[\widetilde{\widehat{H}}_{1}(t), \tilde{\hat{\rho}}(t)\right]=\tilde{L}(t) \tilde{\hat{\rho}}(t)
$$

в котором супероператор $\tilde{L}(t)$ определен как

$$
\tilde{L}(t) \cdot=-\frac{i}{\hbar}\left[\widetilde{\widehat{H}}_{1}(t), \bullet\right] .
$$

Введем пару произвольных проекционных операторов $\mathcal{P}$ и $\mathcal{Q}$ таких, что

$$
\mathcal{P}^{2}=\mathcal{P}, \quad \mathcal{Q}=1-\mathcal{P}, \quad \mathcal{P} \mathcal{Q}=0 .
$$

Тогда имеет место тождество

$$
\tilde{\hat{\rho}}(t)=\mathcal{P} \tilde{\hat{\rho}}(t)+(1-\mathcal{P}) \tilde{\hat{\rho}}(t)=\mathcal{P} \tilde{\hat{\rho}}(t)+\mathcal{Q} \tilde{\hat{\rho}}(t)=\tilde{\hat{\rho}}_{1}(t)+\tilde{\hat{\rho}}_{2}(t),
$$

в котором $\tilde{\hat{\rho}}_{1}(t)$ обозначает релевантную, а $\tilde{\hat{\rho}}_{2}(t)$ - иррелевантную часть статистического оператора $\tilde{\hat{\rho}}(t)$, а из уравнения (42) следует система уравнений для этих частей

$$
\begin{aligned}
& \frac{\partial}{\partial t} \mathcal{P} \tilde{\hat{\rho}}(t)=\frac{\partial}{\partial t} \tilde{\hat{\rho}}_{1}(t)=\mathcal{P} \tilde{L}(t)\left(\tilde{\hat{\rho}}_{1}(t)+\tilde{\hat{\rho}}_{2}(t)\right), \\
& \frac{\partial}{\partial t} \mathcal{Q} \tilde{\hat{\rho}}(t)=\frac{\partial}{\partial t} \tilde{\hat{\rho}}_{2}(t)=\mathcal{Q} \tilde{L}(t)\left(\tilde{\hat{\rho}}_{1}(t)+\tilde{\hat{\rho}}_{2}(t)\right) .
\end{aligned}
$$

Формальное интегрирование второго из этих уравнений дает следующее выражение для иррелевантной части:

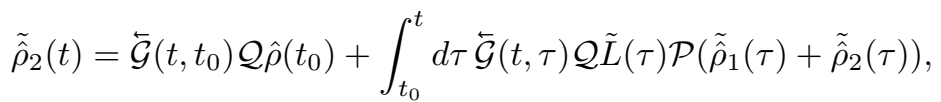

в котором пропагатор

удовлетворяет уравнению

$$
\stackrel{\leftarrow}{\mathcal{G}}(t, \tau)=\stackrel{\leftarrow}{T} \exp \left[\int_{\tau}^{t} d t^{\prime} \mathcal{Q} \tilde{L}\left(t^{\prime}\right)\right]
$$

$$
\frac{\partial \overleftarrow{\mathcal{G}}(t, \tau)}{\partial t}=\mathcal{Q} \tilde{L}(t) \overleftarrow{\mathcal{G}}(t, \tau), \quad \overleftarrow{\mathcal{G}}(\tau, \tau)=1
$$

Подставляя решение (48) в уравнение (46), получаем замкнутое уравнение Накаджимы-Цванцига для релевантной части статистического оператора

$$
\frac{\partial}{\partial t} \tilde{\hat{\rho}}_{1}(t)=\mathcal{P} \tilde{L}(t) \overleftarrow{\mathcal{G}}\left(t, t_{0}\right) \mathcal{Q} \hat{\rho}\left(t_{0}\right)+\mathcal{P} \tilde{L}(t) \mathcal{P} \tilde{\hat{\rho}}_{1}(t)+\int_{t_{0}}^{t} d \tau \mathcal{P} \tilde{L}(t) \overleftarrow{\mathcal{G}}(t, \tau) \mathcal{Q} \tilde{L}(\tau) \mathcal{P} \tilde{\hat{\rho}}_{1}(\tau)
$$

с начальным условием

$$
\tilde{\hat{\rho}}\left(t_{0}\right)=\hat{\rho}\left(t_{0}\right) .
$$

Как обычно, практически всегда можно обеспечить выполнение условий

$$
\mathcal{P} \tilde{L}(t) \mathcal{P}=0, \quad \mathcal{Q} \hat{\rho}\left(t_{0}\right)=0,
$$

в результате чего уравнение (51) существенно упрощается и принимает вид

$$
\frac{\partial}{\partial t} \tilde{\hat{\rho}}_{1}(t)=\int_{t_{0}}^{t} d \tau \mathcal{P} \tilde{L}(t) \stackrel{\leftarrow}{\mathcal{G}}(t, \tau) \mathcal{Q} \tilde{L}(\tau) \mathcal{P} \tilde{\hat{\rho}}_{1}(\tau)
$$




\section{2. Неконволютивное управляющее уравнение для релевантной части} статистического оператора. Уравнение Накаджимы-Цванцига является нелокальным по времени интегро-дифференциальным уравнением, интегральное ядро которого содержит память о состояниях квантовой системы во все моменты времени, предшествующие моменту времени $t$. Существует, однако, альтернативный вариант метода проекционного оператора [5], [11], [12], который позволяет получить эквивалентное чисто дифференциальное, неконволютивное уравнение для релевантной части статистического оператора, которое, будучи локальным по времени, сохраняет тем не менее память об эволюции системы в прошлом. Перепишем уравнение (48) для иррелевантной части в виде

$$
\tilde{\hat{\rho}}_{2}(t)=\overleftarrow{\mathcal{G}}\left(t, t_{0}\right) \mathcal{Q} \hat{\rho}\left(t_{0}\right)+\int_{t_{0}}^{t} d \tau \overleftarrow{\mathcal{G}}(t, \tau) \mathcal{Q} \tilde{L}(\tau) \mathcal{P} \overrightarrow{\mathcal{G}}(t, \tau)\left(\tilde{\hat{\rho}}_{1}(t)+\tilde{\hat{\rho}}_{2}(t)\right)
$$

где пропагатор

$$
\overrightarrow{\mathcal{G}}(t, \tau)=\vec{T} \exp \left[-\int_{\tau}^{t} d t^{\prime} \mathcal{Q} \tilde{L}\left(t^{\prime}\right)\right]
$$

позволил выразить полный статистический оператор $\tilde{\hat{\rho}}(\tau)$ через $\tilde{\hat{\rho}}(t)$ как

$$
\tilde{\hat{\rho}}(\tau)=\overrightarrow{\mathcal{G}}(t, \tau) \tilde{\hat{\rho}}(t)
$$

и избавиться от нелокальности по времени. Введем оператор

$$
\Sigma(t)=\int_{t_{0}}^{t} d \tau \overleftarrow{\mathcal{G}}(t, \tau) \mathcal{Q} \tilde{L}(\tau) \mathcal{P} \overrightarrow{\mathcal{G}}(t, \tau)
$$

который дает возможность преобразовать уравнение (55) к виду

$$
[1-\Sigma(t)] \tilde{\hat{\rho}}_{2}(t)=\overleftarrow{\mathcal{G}}\left(t, t_{0}\right) \mathcal{Q} \hat{\rho}\left(t_{0}\right)+\Sigma(t) \tilde{\hat{\rho}}_{1}(t) .
$$

В предположении, что существует обратный оператор $[1-\Sigma(t)]^{-1}$, иррелевантная часть статистического оператора представима в виде

$$
\tilde{\hat{\rho}}_{2}(t)=[1-\Sigma(t)]^{-1} \overleftarrow{\mathcal{G}}\left(t, t_{0}\right) \mathcal{Q} \hat{\rho}\left(t_{0}\right)+[1-\Sigma(t)]^{-1} \Sigma(t) \tilde{\hat{\rho}}_{1}(t) .
$$

Предположение о существовании обратного оператора $[1-\Sigma(t)]^{-1}$ является основной проблемой обоснования применимости метода неконволютивного управляющего уравнения, однако его можно строго доказать (см. книгу [5]) для достаточно широкого класса задач по крайней мере при условии достаточной малости слагаемого $\widehat{H}_{1}(t)$ и/или достаточно малого временного интервала $t-t_{0}$. Подставляя выражение (60) для иррелевантной части в уравнение (51) для релевантной части, получаем замкнутое локальное по времени уравнение для релевантной части статистического оператора

$$
\frac{\partial}{\partial t} \tilde{\hat{\rho}}_{1}(t)=\mathcal{K}(t) \tilde{\hat{\rho}}_{1}(t)+\mathcal{I}(t) \mathcal{Q} \hat{\rho}\left(t_{0}\right)
$$

где

$$
\begin{aligned}
\mathcal{K}(t) & =\mathcal{P} \tilde{L}(t)[1-\Sigma(t)]^{-1} \mathcal{P}, \\
\mathcal{I}(t) & =\mathcal{P} \tilde{L}(t)[1-\Sigma(t)]^{-1} \overleftarrow{\mathcal{G}}\left(t_{0}, t\right) \mathcal{Q} .
\end{aligned}
$$

Выполнение условия $\mathcal{Q} \hat{\rho}\left(t_{0}\right)=0$ позволяет свести это уравнение к уравнению

$$
\frac{\partial}{\partial t} \tilde{\hat{\rho}}_{1}(t)=\mathcal{K}(t) \tilde{\hat{\rho}}_{1}(t)
$$


3.3. Аппроксимация Борна-Маркова. Во втором порядке по оператору $\widehat{H}_{1}(t)$ уравнение (54) имеет вид

$$
\frac{\partial}{\partial t} \tilde{\hat{\rho}}_{1}(t)=\int_{t_{0}}^{t} d \tau \mathcal{P} \tilde{L}(t) \tilde{L}(\tau) \mathcal{P} \tilde{\hat{\rho}}_{1}(\tau) .
$$

Полагая далее, что

$$
\tilde{\hat{\rho}}(s) \approx \tilde{\hat{\rho}}(t)
$$

приходим к уравнению

$$
\frac{\partial}{\partial t} \tilde{\hat{\rho}}_{1}(t)=\int_{t_{0}}^{t} d \tau \mathcal{P} \tilde{L}(t) \tilde{L}(\tau) \mathcal{P} \tilde{\hat{\rho}}_{1}(t)
$$

в приближении Борна-Маркова. Можно показать посредством формального разложения оператора $[1-\Sigma(t)]^{-1}$ в ряд по степеням $\widehat{H}_{1}(t)$ (см. книгу [5]), что при выполнении условия $\mathcal{P} \tilde{L}(t) \mathcal{P}=0$ уравнение $(64)$ полностью совпадает с уравнением $(67)$ во втором порядке по $\widehat{H}_{1}(t)$, причем при этом не требуется осуществлять аппроксимацию (66) по причине изначальной локальности по времени уравнения (64).

\section{4. МЕТОД ПРОЕКЦИОННОГО ОПЕРАТОРА ДЛЯ $N$-УРОВНЕВОЙ КВАНТОВОЙ СИСТЕМЫ В ТЕРМИНАХ АЛГЕБРЫ $S U(N)$}

4.1. Управляющие уравнения для $N$-уровневой квантовой системы, взаимодействующей с внешними полями и окружением. Предположим теперь, что неравновесная квантовая $N$-уровневая система $(S)$, описываемая гамильтонианом (1), взаимодействует посредством оператора $\widehat{H}_{S B}$ не только с внешними полями, но также и с другой квантовой системой $(B)$, описываемой гамильтонианом $\widehat{H}_{B}$, которая настолько велика, что это взаимодействие практически не выводит ее из состояния теплового равновесия, что позволяет этой системе играть роль термостата. Гамильтониан полной системы $(S+B)$ имеет вид

$$
\widehat{H}(t)=\widehat{H}_{S}(t)+\widehat{H}_{B}+\widehat{H}_{S B} .
$$

Гамильтониан вида (68) типичен для чрезвычайно широкого круга реальных проблем в различных областях теоретической и прикладной физики. Так как нас интересует только эволюция динамических переменных, относящихся к системе $(\mathrm{S})$, то нам достаточно получить замкнутое уравнение для релевантной части статистического оператора

$$
\hat{\rho}_{S}(t)=\operatorname{Sp}_{B}\{\hat{\rho}(t)\},
$$

где след $\mathrm{Sp}_{B}$ берется по квантовым состояниям системы-термостата $(B)$. Мы считаем, что состояние термостата описывается постоянным во времени статистическим оператором $\hat{\rho}_{B}$ :

$$
\hat{\rho}_{B}(t)=\operatorname{Sp}_{S}\{\hat{\rho}(t)\} \equiv \hat{\rho}_{B} .
$$

Если сформулировать естественное с точки зрения типичных начальных условий реального эксперимента предположение о том, что в начальный момент времени состояние $N$-уровневой подсистемы $(S)$ было приготовлено таким образом, что корреляции между этой системой и термостатом отсутствовали, т. е.

$$
\hat{\rho}\left(t_{0}\right)=\hat{\rho}_{S}\left(t_{0}\right) \hat{\rho}_{B}\left(t_{0}\right)=\hat{\rho}_{S}\left(t_{0}\right) \hat{\rho}_{B}
$$


то целесообразно выбрать проекционный оператор $\mathcal{P}$ в виде

$$
\mathcal{P}=\hat{\rho}_{B} \operatorname{Sp}_{B}, \quad \text { так что }(1-\mathcal{P}) \hat{\rho}\left(t_{0}\right)=0 .
$$

Без ограничения общности можно считать, что

$$
\operatorname{Sp}_{B}\left\{\widehat{H}_{S B} \hat{\rho}_{B}\right\}=0,
$$

так как если это условие изначально не выполняется, то всегда можно переопределить гамильтонианы $H_{S}(t)$ и $H_{S B}$ как

$$
\widehat{H}_{S}(t) \rightarrow \widehat{H}_{S}(t)+\operatorname{Sp}_{B}\left\{\widehat{H}_{S B} \hat{\rho}_{B}\right\}, \quad \widehat{H}_{S B} \rightarrow \widehat{H}_{S B}-\operatorname{Sp}_{B}\left\{\widehat{H}_{S_{B}} \hat{\rho}_{B}\right\},
$$

так что их сумма останется неизменной при одновременном выполнении условия (73), из которого следует соотношение

$$
\mathcal{P} L_{S B} \mathcal{P}=0, \quad L_{S B} \bullet=-\frac{i}{\hbar}\left[\widehat{H}_{S B}, \bullet\right]
$$

Заметим, что гамильтониан взаимодействия между $N$-уровневой системой и термостатом можно представить в общей форме

$$
\widehat{H}_{S B}=\sum_{k=1}^{N^{2}-1} \widehat{E}_{k} \hat{s}_{k}+\widehat{E}_{0} \hat{I},
$$

где относящиеся к термостату операторы $\widehat{E}_{k}$ определены как

$$
\widehat{E}_{k}=\frac{1}{2} \operatorname{Sp}_{S}\left\{\widehat{H}_{S B} \hat{s}_{k}\right\}, \quad \widehat{E}_{0}=N^{-1} \operatorname{Sp}_{S}\left\{\widehat{H}_{S B}\right\}
$$

в полной аналогии с выражениями (13) и (17). Второе слагаемое не содержит операторов $\hat{s}_{k}$ и обычно равно нулю в практически полезных моделях. В противном случае можно считать, что оно должно быть включено в состав гамильтониана термостата $H_{B}$ в гамильтониане (68). Впрочем, как будет видно далее, в любом случае это слагаемое не приводит к существенным изменениям в практически полезных приближенных уравнениях (79), (82), так как вносит в них поправки более высокого порядка по взаимодействию с термостатом, которыми можно пренебречь. Тогда, полагая в гамильтониане (36)

$$
\widehat{H}_{0}(t)=\widehat{H}_{S}(t)+\widehat{H}_{B}, \quad \widehat{H}_{1}(t)=\widehat{H}_{S B},
$$

из уравнения (65) для релевантной части статистического оператора получаем конволютивное уравнение во втором порядке по оператору взаимодействия с термостатом

$$
\begin{aligned}
& \frac{\partial}{\partial t} \tilde{\hat{\rho}}_{S}(t)= \\
& =-\frac{1}{\hbar^{2}} \sum_{n, m=1}^{N^{2}-1} \int_{t_{0}}^{t} d t^{\prime}\left[\tilde{\hat{s}}_{n}\left(t, t_{0}\right), \tilde{\hat{s}}_{m}\left(t^{\prime}, t_{0}\right) \tilde{\hat{\rho}}_{S}\left(t^{\prime}\right)\right] \operatorname{Sp}_{B}\left\{\widetilde{\widehat{E}}_{n}\left(t, t_{0}\right) \widetilde{\widehat{E}}_{m}\left(t^{\prime}, t_{0}\right) \hat{\rho}_{B}\left(t_{0}\right)\right\}- \\
& \quad-\frac{1}{\hbar^{2}} \sum_{n, m=1}^{N^{2}-1} \int_{t_{0}}^{t} d t^{\prime}\left[\tilde{\hat{s}}_{n}\left(t, t_{0}\right), \tilde{\hat{\rho}}_{S}\left(t^{\prime}\right) \tilde{\hat{s}}_{m}\left(t^{\prime}, t_{0}\right)\right] \operatorname{Sp}_{B}\left\{\widetilde{\widehat{E}}_{m}\left(t^{\prime}, t_{0}\right) \widetilde{\widehat{E}}_{n}\left(t, t_{0}\right) \hat{\rho}_{B}\left(t_{0}\right)\right\}
\end{aligned}
$$


в котором статистический оператор $\tilde{\hat{\rho}}_{S}(t)$ и все прочие операторы фигурируют в представлении взаимодействия вида $(37),(38)$ с гамильтонианом

$$
H_{0}(t)=H_{S}(t)+H_{B},
$$

т. е. операторы $\tilde{\hat{s}}_{n}(t)$ имеют вид

$$
\tilde{\hat{s}}_{n}\left(t, t_{0}\right)=U^{\dagger}\left(t, t_{0}\right) \hat{s}_{m} U\left(t, t_{0}\right)=\sum_{l=1}^{N^{2}-1} \widetilde{C}_{n l}\left(t, t_{0}\right) \hat{s}_{l},
$$

где коэффициенты $\widetilde{C}_{n k}(t)$ вычисляются по формулам $(21)$ или $(22)$ для эволюции под воздействием гамильтониана (80).

Обычно предполагается, что состояние термостата не изменяется при взаимодействии его с подсистемой $S$, т. е. что корреляционные функции термостата быстро релаксируют за время $t-t^{\prime} \leqslant \tau_{B}$, где $\tau_{B}$ - характерное время корреляции, зависящее от структуры и типа термостата. При $t-t^{\prime} \gg \tau_{B}$ корреляции пренебрежимо малы, таким образом, время $\tau_{B}$ задает временной масштаб, на котором сохраняется память о взаимодействии. Тогда интеграл в уравнении (79) существенно отличается от нуля только для $t-t^{\prime} \leqslant \tau_{B}$, т. е. влияние $\rho_{S}\left(t^{\prime}\right)$ на $\dot{\rho}_{S}(t)$ пренебрежимо мало за пределами этого интервала. Поэтому можно считать, что $\rho_{S}\left(t^{\prime}\right) \approx \rho_{S}(t)$, что обычно называют первым приближением Маркова. В этом приближении уравнение (79) сводится к частному случаю неконволютивного уравнения (67), известному как уравнение Редфилда [13]:

$$
\begin{aligned}
\frac{\partial}{\partial t} \tilde{\hat{\rho}}_{S}(t) & = \\
= & -\frac{1}{\hbar^{2}} \sum_{n, m=1}^{N^{2}-1} \int_{t_{0}}^{t} d t^{\prime}\left[\tilde{\hat{s}}_{n}\left(t, t_{0}\right), \tilde{\hat{s}}_{m}\left(t^{\prime}, t_{0}\right) \tilde{\hat{\rho}}_{S}(t)\right] \operatorname{Tr}_{B}\left\{\widetilde{\widehat{E}}_{n}\left(t, t_{0}\right) \widetilde{\widehat{E}}_{m}\left(t^{\prime}, t_{0}\right) \hat{\rho}_{B}\left(t_{0}\right)\right\}- \\
& -\frac{1}{\hbar^{2}} \sum_{n, m=1}^{N^{2}-1} \int_{t_{0}}^{t} d t^{\prime}\left[\tilde{\hat{s}}_{n}\left(t, t_{0}\right), \tilde{\hat{\rho}}_{S}(t) \tilde{\hat{s}}_{m}\left(t^{\prime}, t_{0}\right)\right] \operatorname{Tr}_{B}\left\{\widetilde{\widehat{E}}_{m}\left(t^{\prime}, t_{0}\right) \widetilde{\widehat{E}}_{n}\left(t, t_{0}\right) \hat{\rho}_{B}\left(t_{0}\right)\right\}
\end{aligned}
$$

Если ввести некоторое характерное время изменения параметров системы $1 / \gamma$, то приближение Маркова имеет смысл, если $\tau_{B} \ll 1 / \gamma$. Это означает, что полученное уравнение не может описывать динамические процессы в системе, характерные времена которых соизмеримы с временем корреляции $\tau_{B}$. Этот факт позволяет осуществить второе приближение Маркова, которое заключается в замене переменных $t^{\prime}=t-\tau$ в уравнении (82) и последующей замене верхнего предела интегрирования на $t-t_{0} \rightarrow \infty$. Данное приближение приводит к уравнению, которое описывает марковскую эволюцию системы. Согласно выражению (10), будем искать решение для $\tilde{\hat{\rho}}_{S}(t)$ в виде

$$
\tilde{\hat{\rho}}(t)=N^{-1} \hat{I}+\frac{1}{2} \sum_{j=1}^{N^{2}-1} \widetilde{S}_{i}(t) \hat{s}_{i} .
$$

Подставляя разложения (81) и (83) в левую и правую части уравнений (79) и (82) и собирая коэффициенты при операторах $\hat{s}_{k}$, получаем соответственно систему 
интегро-дифференциальных или дифференциальных уравнений для коэффициентов $\widetilde{S}_{k}(t)$, а именно: из уравнения $(79)$ следует, что

$$
\frac{\partial}{\partial t} \widetilde{S}_{i}(t)=\sum_{k=1}^{N^{2}-1} \int_{t_{0}}^{t} d t^{\prime} \tilde{A}_{i k}\left(t, t^{\prime}, t_{0}\right) \widetilde{S}_{k}\left(t^{\prime}\right)+\int_{t_{0}}^{t} d t^{\prime} \tilde{I}_{i}\left(t, t^{\prime}, t_{0}\right),
$$

а из уравнения (82) следует, что

$$
\frac{\partial}{\partial t} \widetilde{S}_{i}(t)=\sum_{k=1}^{N^{2}-1} \int_{t_{0}}^{t} d t^{\prime} \tilde{A}_{i k}\left(t, t^{\prime}, t_{0}\right) \widetilde{S}_{k}(t)+\int_{t_{0}}^{t} d t^{\prime} \tilde{I}_{i}\left(t, t^{\prime}, t_{0}\right)
$$

где

$$
\begin{aligned}
\tilde{A}_{i k}\left(t, t^{\prime}, t_{0}\right)= & -\frac{1}{4 \hbar^{2}} \sum_{n, m}^{N^{2}-1}\left[\widetilde{C}_{n m k}^{(1) i}\left(t, t^{\prime}, t_{0}\right) \operatorname{Sp}_{B}\left\{\widetilde{E}_{n}(t) \widetilde{\widehat{E}}_{m}\left(t^{\prime}\right) \hat{\rho}_{B}\left(t_{0}\right)\right\}\right]- \\
& -\frac{1}{4 \hbar^{2}} \sum_{n, m}^{N^{2}-1}\left[\widetilde{C}_{n m k}^{(2) i}\left(t, t^{\prime}, t_{0}\right) \operatorname{Sp}_{B}\left\{\widetilde{E}_{m}\left(t^{\prime}\right){\widetilde{E_{n}}}_{n}(t) \hat{\rho}_{B}\left(t_{0}\right)\right\}\right] \\
\widetilde{C}_{n m k}^{(1) i}\left(t, t^{\prime}, t_{0}\right)= & \sum_{l, p=1}^{N^{2}-1} \widetilde{C}_{n l}\left(t, t_{0}\right) \widetilde{C}_{m p}\left(t^{\prime}, t_{0}\right) \operatorname{Sp}_{S}\left\{\left[\hat{s}_{l}, \hat{s}_{p} \hat{s}_{k}\right] \hat{s}_{i}\right\} \\
\widetilde{C}_{n m k}^{(2) i}\left(t, t^{\prime}, t_{0}\right)= & \sum_{l, p=1}^{N^{2}-1} \widetilde{C}_{n l}\left(t, t_{0}\right) \widetilde{C}_{m p}\left(t^{\prime}, t_{0}\right) \operatorname{Sp}_{S}\left\{\left[\hat{s}_{l}, \hat{s}_{k} \hat{s}_{p}\right] \hat{s}_{i}\right\} \\
\tilde{I}_{i}\left(t, t^{\prime}, t_{0}\right)= & -\frac{1}{2 N \hbar^{2}} \sum_{n, m}^{N^{2}-1}\left[\widetilde{C}_{n m}^{i}\left(t, t^{\prime}, t_{0}\right) \operatorname{Sp}_{B}\left\{\widetilde{\widehat{E}}_{n}(t) \widetilde{\widehat{E}}_{m}\left(t^{\prime}\right) \hat{\rho}_{B}\left(t_{0}\right)\right\}\right]- \\
& -\frac{1}{2 N \hbar^{2}} \sum_{n, m}^{N^{2}-1}\left[\widetilde{C}_{n m}^{i}\left(t, t^{\prime}, t_{0}\right) \operatorname{Sp}_{B}\left\{\widetilde{\widehat{E}}_{m}\left(t^{\prime}\right) \widetilde{\widehat{E}}_{n}(t) \hat{\rho}_{B}\left(t_{0}\right)\right\}\right] \\
& \\
\widetilde{C}_{n m}^{i}\left(t, t^{\prime}, t_{0}\right)= & 4 i \sum_{l, p=1}^{N^{2}-1} \widetilde{C}_{n l}\left(t, t_{0}\right) \widetilde{C}_{m p}\left(t, t^{\prime}, t_{0}\right) f_{i l p} .
\end{aligned}
$$

\section{2. Полиномиальная аппроксимация для статистического оператора} $N$-уровневой квантовой системы. Перейдем теперь к представлению Шредингера в уравнении (79):

$$
\begin{aligned}
& \frac{\partial}{\partial t} \tilde{\hat{\rho}}_{S}(t)=-\frac{i}{\hbar}\left[\widehat{H}_{S}(t), \hat{\rho}_{S}(t)\right]- \\
& \quad-\frac{1}{\hbar^{2}} \sum_{n, m=1}^{N^{2}-1} \int_{t_{0}}^{t} d t^{\prime}\left[\hat{s}_{n}, \tilde{\hat{s}}_{m}\left(t^{\prime}, t\right) U_{0}\left(t, t^{\prime}\right) \hat{\rho}_{S}\left(t^{\prime}\right) U_{0}^{\dagger}\left(t, t^{\prime}\right)\right] \operatorname{Sp}_{B}\left\{\widetilde{\widehat{E}}_{n}\left(t, t_{0}\right) \widetilde{\widehat{E}}_{m}\left(t^{\prime}, t_{0}\right) \hat{\rho}_{B}\left(t_{0}\right)\right\}- \\
& \quad-\frac{1}{\hbar^{2}} \sum_{n, m=1}^{N^{2}-1} \int_{t_{0}}^{t} d t^{\prime}\left[\hat{s}_{n}, U_{0}\left(t, t^{\prime}\right) \hat{\rho}_{S}\left(t^{\prime}\right) U_{0}^{\dagger}\left(t, t^{\prime}\right) \tilde{\hat{s}}_{m}\left(t^{\prime}, t\right)\right] \operatorname{Sp}_{B}\left\{\widetilde{\widehat{E}}_{m}\left(t^{\prime}, t_{0}\right) \widetilde{\widehat{E}}_{n}\left(t, t_{0}\right) \hat{\rho}_{B}\left(t_{0}\right)\right\},
\end{aligned}
$$


где

$$
\tilde{\hat{s}}_{m}\left(t^{\prime}, t\right)=U^{\dagger}\left(t^{\prime}, t\right) \hat{s}_{m} U\left(t^{\prime}, t\right)=\sum_{l=1}^{N^{2}-1} \widetilde{C}_{m l}\left(t^{\prime}, t\right) \hat{s}_{l} .
$$

Ввиду слабого взаимодействия с термостатом обычно предполагают, что

$$
U_{0}\left(t, t^{\prime}\right) \hat{\rho}_{S}\left(t^{\prime}\right) U_{0}^{\dagger}\left(t, t^{\prime}\right) \approx \hat{\rho}_{S}(t),
$$

в результате чего уравнение (91) становится чисто дифференциальным, но можно сохранить интегро-дифференциальное уравнение, если вместо приближения (93) использовать точное соотношение

$$
\begin{aligned}
& U_{0}\left(t, t^{\prime}\right) \hat{\rho}_{S}\left(t^{\prime}\right) U_{0}^{\dagger}\left(t, t^{\prime}\right)=N^{-1} \hat{I}+\frac{1}{2} \sum_{k=1}^{N^{2}-1} S_{k}\left(t^{\prime}\right) U_{0}\left(t, t^{\prime}\right) \hat{s}_{k} U_{0}^{\dagger}\left(t, t^{\prime}\right)= \\
& =N^{-1} \hat{I}+\frac{1}{2} \sum_{k=1}^{N^{2}-1} S_{k}\left(t^{\prime}\right) \tilde{\hat{s}}_{k}\left(t^{\prime}, t\right)=N^{-1} \hat{I}+\frac{1}{2} \sum_{k, l=1}^{N^{2}-1} S_{k}\left(t^{\prime}\right) \widetilde{C}_{k l}\left(t^{\prime}, t\right) \hat{s}_{l} .
\end{aligned}
$$

Рассмотрим практически важный случай, когда $N$-уровневая система взаимодействует одновременно с внешними классическими полями и термостатом, но при этом либо можно устранить явную временную зависимость в слагаемых, описывающих взаимодействие с внешними полями при помощи подходящего унитарного преобразования гамильтониана (68), либо функции

$$
f_{i k}(t)=\sum_{j=1}^{N^{2}-1} f_{i j k} \Gamma_{j}(t)
$$

в уравнениях (18) изменяются незначительно на интервале времени $t-t_{0}$ и их можно аппроксимировать некоторыми постоянными средними значениями на этом интервале. Известным примером вышеупомянутого унитарного преобразования в задачах квантовой оптики является переход к так называемой вращающейся системе координат (rotating frame transformation). Тогда из уравнения (91) следует система уравнений для коэффициентов $S_{k}(t)$, содержащих интегральные слагаемые типа свертки:

$$
\frac{\partial}{\partial t} S_{i}(t)=\sum_{j, k=1}^{N^{2}-1} f_{i j k} \Gamma_{j} S_{k}(t)+\sum_{k=1}^{N^{2}-1} \int_{0}^{t} d t^{\prime} A_{i k}\left(t-t^{\prime}\right) S_{k}\left(t^{\prime}\right)+\int_{t_{0}}^{t} d t^{\prime} I_{i}\left(t-t^{\prime}, 0\right),
$$

где

$$
\begin{aligned}
A_{i k}\left(t-t^{\prime}, 0\right)= & -\frac{1}{4 \hbar^{2}} \sum_{n, m}^{N^{2}-1}\left[C_{n m k}^{(1) i}\left(t-t^{\prime}, 0\right) \operatorname{Sp}_{B}\left\{\widetilde{\widehat{E}}_{n}(0) \widetilde{\widehat{E}}_{m}\left(t-t^{\prime}\right) \hat{\rho}_{B}(0)\right\}\right]- \\
& -\frac{1}{4 \hbar^{2}} \sum_{n, m}^{N^{2}-1}\left[C_{n m k}^{(2) i}\left(t-t^{\prime}, 0\right) \operatorname{Sp}_{B}\left\{\widetilde{\widehat{E}}_{m}\left(t-t^{\prime}\right) \widetilde{\widehat{E}}_{n}(0) \hat{\rho}_{B}(0)\right\}\right], \\
C_{n m k}^{(1) i}\left(t-t^{\prime}, 0\right)= & \sum_{l, p=1}^{N^{2}-1} C_{m p}\left(t^{\prime}-t, 0\right) C_{k l}\left(t^{\prime}-t, 0\right) \operatorname{Sp}_{S}\left\{\left[\hat{s}_{n}, \hat{s}_{p} \hat{s}_{l}\right] \hat{s}_{i}\right\},
\end{aligned}
$$




$$
\begin{aligned}
C_{n m k}^{(2) i}\left(t-t^{\prime}, 0\right)= & \sum_{l, p=1}^{N^{2}-1} C_{m p}\left(t^{\prime}-t, 0\right) C_{k l}\left(t^{\prime}-t, 0\right) \operatorname{Sp}_{S}\left\{\left[\hat{s}_{n}, \hat{s}_{l} \hat{s}_{p}\right] \hat{s}_{i}\right\}, \\
I_{i}\left(t-t^{\prime}, 0\right)= & -\frac{1}{2 N \hbar^{2}} \sum_{n, m}^{N^{2}-1}\left[C_{n m}^{i}\left(t-t^{\prime}, 0\right) \operatorname{Sp}_{B}\left\{\widetilde{\widehat{E}}_{n}(0) \widetilde{\widehat{E}}_{m}\left(t-t^{\prime}\right) \hat{\rho}_{B}(0)\right\}\right]- \\
& -\frac{1}{2 N \hbar^{2}} \sum_{n, m}^{N^{2}-1}\left[C_{n m}^{i}\left(t-t^{\prime}\right) \operatorname{Sp}_{B}\left\{\widetilde{\widehat{E}}_{m}\left(t-t^{\prime}, 0\right) \widetilde{\widehat{E}}_{n}(0) \hat{\rho}_{B}(0)\right\}\right], \\
C_{n m}^{i}\left(t-t^{\prime}, 0\right)= & 4 i \sum_{p=1}^{N^{2}-1} C_{m p}\left(t^{\prime}-t, 0\right) f_{i n p} .
\end{aligned}
$$

В случае слабого взаимодействия с внешними полями все эти коэффициенты можно вычислить в пренебрежении влиянием внешних полей в гамильтониане (1). При выводе этих уравнений предполагалось для удобства и без потери общности, что $t_{0}=0$, а также что термостат находится в термодинамически равновесном состоянии, описываемом статистическим оператором $\hat{\rho}_{B}=e^{-\beta \widehat{H}_{B}} / \operatorname{Sp}_{B}\left\{e^{-\beta \widehat{H}_{B}}\right\}$, поэтому равновесные корреляционные функции от операторных переменных термостата зависят только от разности временных аргументов $t-t^{\prime}$.

Немарковское уравнение (96) для статистического оператора с учетом эффекта памяти можно преобразовать от интегро-дифференциального к чисто алгебраическому виду с использованием разложения по полиномам Лагерра, которые определены выражением

$$
L_{n}(x)=\sum_{k=0}^{n} \frac{(-1)^{k}}{k !} C_{n}^{k} x^{k}=\frac{e^{x}}{n !} \frac{d^{n}}{d x^{n}}\left(x^{n} e^{-x}\right)
$$

где $C_{n}^{k}$ - биноминальные коэффициенты, и ортогональны в смысле скалярного произведения

$$
\left(L_{n}(x), L_{m}(x)\right)=\int_{0}^{\infty} d x e^{-x} L_{n}(x) L_{m}(x)=\delta_{n m} .
$$

Для полиномов Лагерра имеют место важные соотношения

$$
\begin{aligned}
\frac{\partial}{\partial x} L_{n}(x) & =-\sum_{m=0}^{n-1} L_{m}(x) \\
\int_{0}^{x} d x^{\prime} L_{n}\left(x-x^{\prime}\right) L_{m}\left(x^{\prime}\right) & =L_{n+m}(x)-L_{n+m+1}(x) .
\end{aligned}
$$

При $n>1$ полиномы Лагерра можно также определить рекуррентной формулой

$$
L_{n+1}(x)=\frac{1}{n+1}\left[(2 n+1-x) L_{n}(x)-n L_{n-1}(x)\right]
$$

предопределив первые два полинома в виде $L_{0}(x)=1, L_{1}(x)=1-x$, что делает возможным практическое вычисление значений полиномов высокого порядка. Соотношение (105) особенно полезно, так как оно позволяет избавиться от нелокальности по времени в интегро-дифференциальном уравнении (96) и перейти от 
системы интегро-дифференциальных уравнений для коэффициентов $S_{j}(t)$ к бесконечной системе обыкновенных линейных уравнений для коэффициентов разложения функций $S_{j}(t)$ по полиномам Лагерра

$$
S_{i}\left(x t_{\mathrm{c}}\right)=\sum_{n=0}^{\infty} S_{i}^{(n)} L_{n}(x)
$$

где

$$
S_{i}^{(n)}=\int_{0}^{\infty} d x e^{-x} L_{n}(x) S_{i}\left(x t_{\mathrm{c}}\right)
$$

a $x=t / t_{\mathrm{c}}$ обезразмеренное время, причем $t_{\mathrm{c}}$ есть некоторый произвольный временной масштаб, численное значение которого выбирается исходя из параметров исследуемой физической системы в каждом конкретном случае. Важно отметить также, что

$$
S_{i}(t=0)=\sum_{n=0}^{\infty} S_{i}^{(n)},
$$

так как $L_{n}(x=0)=1$ для всех $n$. В результате из уравнения (96) следуют рекуррентные соотношения для коэффициентов разложения $S_{i}^{(n)}$

$$
\begin{aligned}
& -\left(\sum_{j, k=1}^{N^{2}-1} f_{i j k} \Gamma_{j}+t_{\mathrm{c}}^{2} A_{i k}^{(0)}\right) S_{k}^{(n)}= \\
& =S_{i}(t=0)-\sum_{m=0}^{n-1}\left(S_{i}^{(m)}+t_{\mathrm{c}}^{2} \sum_{k=1}^{N^{2}-1}\left\{A_{i k}^{(n-m)}-A_{i k}^{(n-m-1)}\right\} S_{k}^{(m)}\right)+t_{\mathrm{c}} I_{i}^{(n)},
\end{aligned}
$$

где

$$
A_{i k}^{(n)}=\int_{0}^{\infty} d x e^{-x} L_{n}(x) A_{i k}\left(x t_{\mathrm{c}}, 0\right), \quad I_{i}^{(n)}=\int_{0}^{\infty} d x e^{-x} L_{n}(x) I_{i}\left(x t_{\mathrm{c}}, 0\right) .
$$

Очевидно, что полиномиальная аппроксимация конечного порядка $n$ для статистического оператора $\hat{\rho}_{S}(t)$ способна обеспечить заданную точность аппроксимации лишь для некоторого достаточно короткого временного интервала $\Delta t=t-t_{0}$, причем необходимый порядок аппроксимации и длина интервала взаимозависимы. Однако если необходимо получить решение неконволютивного уравнения (96) на произвольном интервале $t-t_{0}$, этот интервал можно разбить на множество интервалов длиной $\Delta t$, на каждом из которых внешние поля можно считать постоянными, и статистический оператор можно вычислить с необходимой точностью при помощи полиномиальной аппроксимации (107) соответствующего порядка для каждого последующего временного интервала с учетом уже известной эволюции статистического оператора, вычисленной для всех предшествующих интервалов. Подход к анализу уравнения (96), основанный на полиномиальной аппроксимации, может оказаться особенно целесообразным при исследовании эволюции на малых временных масштабах квантовой $N$-уровневой системы, взаимодействующей с внешними классическими полями, характерное время изменения которых соизмеримо с характерным временем затухания корреляционных функций переменных термостата и характерными временами собственных колебаний невозмущенной квантовой системы. 
Погрешность полиномиальной аппроксимации порядка $n$ на некотором временном интервале $\left[t_{1}, t_{2}\right]$ можно оценить, вычисляя оценку величины среднего абсолютного отклонения для коэффициентов $S_{i}^{(n)}$ на этом интервале как функцию $n$ для достаточно большой величины $\Delta n$, а именно:

$$
\Delta S_{i}^{(n)}\left(n, t_{1}, t_{2}\right)=\frac{t_{\mathrm{c}}}{t_{2}-t_{1}} \lim _{\Delta n \rightarrow \infty} \int_{t_{1} / t_{\mathrm{c}}}^{t_{2} / t_{\mathrm{c}}}\left|\sum_{m=n+1}^{n+\Delta n} S_{i}^{(m)} L_{m}(x)\right| d x .
$$

\section{5. ЗАКЛЮЧЕНИЕ}

Результаты проведенного исследования позволяют сделать вывод о том, что использование для наблюдаемых и статистического оператора многоуровневой квантовой системы с произвольным конечным числом квантовых состояний $N$ специального представления в терминах операторной алгебры $S U(N)$ существенно упрощает вычисления не только свободной динамики такой системы, взаимодействующей с произвольными классическими внешними полями различной природы, но и позволяет получить как локальные, так и нелокальные по времени приближенные управляющие уравнения для статистического оператора в удобной для последующего численного анализа форме в общем случае взаимодействия многоуровневой системы не только с внешними детерминированными полями, но и с диссипативным окружением для широкого класса таких взаимодействий. При этом нелокальные по времени уравнения для статистического оператора в представлении Шредингера допускают численное решение методом разложения по системе ортогональных многочленов, в частности полиномов Лагерра, что дает возможность свести задачу решения приближенного интегро-дифференциального уравнения к вычислению коэффициентов разложения статистического оператора по этим полиномам посредством рекуррентных соотношений. Это обстоятельство в совокупности с возможностью вычислять значения полиномов Лагерра высших порядков при помощи рекуррентного соотношения способно обеспечить высокую точность аппроксимации статистического оператора при сравнительно небольшом объеме вычислений. Тот факт, что в представлении алгебры $S U(N)$ эволюция $N$-уровневой системы под воздействием внешних классических полей эффективно сводится к вращению некоторого вещественного вектора в пространстве $N^{2}-1$ измерений, а также положительно сказывается как на объеме вычислений, так и на их точности при численном анализе как собственно динамики такой системы, так и при вычислении зависящих от времени коэффициентов, входящих в состав управляющих уравнений для статистического оператора. Для повышения точности вычисления этих величин целесообразно использовать численные алгоритмы, обеспечивающие ускоренную сходимость при вычислении эволюционных операторов, например методы, предложенные в работе [14]. Отметим, что в процессе вывода как локальных, так и нелокальных по времени приближенных уравнений для статистического оператора не делалось предположения о слабости взаимодействия $N$-уровневой системы с внешними классическими полями, предполагалось лишь слабое взаимодействие этой системы с окружением, взаимодействие же с внешними полями учитывалось точно через вычисление временной зависимости соответствующих коэффициентов в составе интегральных ядер этих уравнений. Предложенный формализм позволяет вычислять и высшие поправки в разложении по взаимодействию с окружением для формально точных уравнений для статистического оператора вида (54) и (64) также без предположения о слабости 
взаимодействия с внешними классическими полями. Численное решение полученных в результате такого разложения приближенных интегро-дифференциальных уравнений высших порядков также можно свести к вычислению коэффициентов разложения статистического оператора по системе полиномов Лагерра или иной подходящей системе ортогональных многочленов посредством соответствующих рекуррентных соотношений. Предложенный формализм естественным образом обобщается на случай явно зависящего от времени взаимодействия квантовой $N$-уровневой системы с окружением. Его также можно адаптировать для вычисления многовременных неравновесных корреляционных функций от наблюдаемых $N$-уровневой квантовой системы, что, однако, составляет предмет отдельного исследования.

\section{Список литературы}

[1] Н. Н. Боголюбов, Н. М. Крылов, “Об уравнениях Фоккера-Планка, которые выводятся в теории возмущений методом, основанным на спектральных свойствах возмущенного гамильтониана", Зап. кафедры матем. физ. Института нелинейной механики АН УССР, 4 (1939), 5-80; Н. Н. Боголюбов, Собрание научных трудов в 12 томах. Статистическая механика, т. 5: Неравновесная статистическая механика. 1939-1980, Наука, М., 2006.

[2] Н. Н. Боголюбов, О некоторых статистических методах в математической физи-

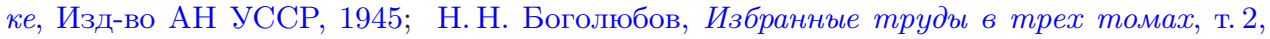
Наукова думка, Киев, 1970.

[3] Н.Н. Боголюбов, Проблемы динамической теории в статистической физике, Гостехиздат, М.-Л., 1946; Н.Н. Боголюбов, Избранные труды в трех томах, т. 2, Наукова думка, Киев, 1970, 99-196.

[4] Д. Н. Зубарев, В. Г. Морозов, Г. Рёпке, Статистическая механика неравновеснъх проиессов, т. 1, 2, Физматлит, М., 2002.

[5] H.-P. Breuer, F. Petruccione, The Theory of Open Quantum Systems, Oxford Univ. Press, Oxford, 2002.

[6] E. Fick, G. Sauermann, The Quantum Statistics of Dynamic Processes, Springer Series in Solid-State Sciences, 86, Springer, Berlin, 1990.

[7] F. T. Hioe, J. H. Eberly, " $N$-level coherence vector and higher conservation laws in quantum optics and quantum mechanics", Phys. Rev. Lett., 47:12 (1981), 838-841.

[8] R. R. Puri, " $S U(m, n)$ coherent states in the bosonic representation and their generation in optical parametric processes", Phys. Rev. A, 50:6 (1994), 5309-5316.

[9] R. R. Puri, Mathematical methods of quantum optics, Springer Series in Optical Sciences, 79, Springer, Berlin, 2001.

[10] R. Zwanzig, "Ensemble method in the theory of irreversibility", J. Chem. Phys., 33:5 (1960), 1338-1341.

[11] F. Shibata, Y. Takahashi, N. Hashitsume, "A generalized stochastic Liouville equation. Non-Markovian versus memoryless master equations", J. Statist. Phys., 17:4 (1977), 171-187.

[12] H.-P. Breuer, B. Kappler, F. Petruccione, "The time-convolutionless projection operator technique in the quantum theory of dissipation and decoherence", Ann. Phys., 291:1 (2001), 36-70.

[13] A. G. Redfield, "On the theory of relaxation processes", IBM J. Res. Dev., 1:1 (1957), 19-31.

[14] M. Suzuki, "Generalized Trotter's formula and systematic approximants of exponential operators and inner derivations with applications to many-body problems", Comm. Math. Phys., 51:2 (1976), 183-190. 